\title{
The Thermal Behaviour of a Cylindrical Air Layer Enclosed between Double Fabric Roof Membranes
}

\author{
Dragan KOSTIC*, Veliborka BOGDANOVIC, Marko ILIC, Miomir VASOV, Vuk MILOSEVIC
}

\begin{abstract}
Objects covered by fabric roof membranes are specific in terms of energy consumption, primarily because of the negligible thickness of the material and its good thermal conduction properties. One of the ways of improving the energy efficiency of these objects is the implementation of a double fabric roof membrane structure with an enclosed non ventilated air layer. This paper analyzes the thermal-insulation behaviour of the air layer enclosed between tensile fabric roof membranes which are used for structural purposes. The compilation of the necessary data was carried out by measuring a real object covered by a double fabric roof membrane, semi-cylindrical in shape. The surface temperatures of each membrane were measured, as were the outside and inside air temperature and the air temperature between the membranes during the summer months. In addition, the solar irradiance during the same period was also measured. The analysis of the measured data took into consideration all the present forms of energy transmittance (convection, conduction and radiation), as well as the shape of the air layer and the thermal-physical specific features of border surfaces. The results of the study indicate that thermal behaviour of a closed semi-cylindrical air layer depends on the real outer conditions in the summer months. A methodological approach to the thermal modelling of these structures has accordingly been proposed.
\end{abstract}

Keywords: air layer resistance; air enclosed between fabric membranes; air layer thermal properties; double membrane structure; thermal behavior in summer months

\section{INTRODUCTION}

Contemporary tendencies in the shaping of roof surfaces, the needs for natural light, the improvement of structural characteristics of fabric material and numeric procedures for analysis and calculation have led to an increase in the popularity of tensile structures for covering public objects, mostly semi-open objects.

The negligibly small thickness of the fabric, ranging from $0.8 \mathrm{~mm}$ to $1.6 \mathrm{~mm}$ and the exceptional core heat conduction of the fabric membranes $0.19 \mathrm{Wm}^{-1} \mathrm{~K}^{-1}$ indicate high losses in the winter, that is, high heat gain in the summer. The transparency of the fabric leads to great energy savings on lighting. The energy influence of the structural fabric is the function of the balance between the decreased need for lighting and increased costs for cooling/heating. The establishment of thermal comfort in closed tensile structures represents a very demanding energy problem, which is especially pronounced in the summer due to increased heat gain due to solar radiation. The prescribed thermal comfort includes an improvement in the thermal-physical properties of the outer fabric layer.

One of the suggestions for the improvement in the thermal-physical properties of the membranes is the addition of a thermal insulator layer to the fabric membrane [1] which greatly diminishes its transparency. The most frequently implemented means of reducing energy consumption is the formation of multiple layers of gaps which can be ventilated, unventilated or filled in with insulating material. In addition, energy savings can be achieved by covering traditional objects with closed tensile structures [2].

Research in the field of thermal properties of structural membranes has still not provided guidelines for energy optimized, closed double structures. The thermal properties of single open tensile structures were studied by He \& Hoyano $[3,4]$. The thermal and optical aspects of closed and semi-closed membrane structures were studied by Elnokali, Chilton \& Wilson [5]. The most thorough research into the behavior of membranes and heat transport was carried out by Harvie [6] who took into consideration solar radiation, transmittance and reflectance, as well as convection and conduction through the structure of the material by applying a dynamic approach.

Numerous studies have been carried out on the behavior of fabric structures in order to meet the needs of the clothing industry for ready-to-wear clothing and the thermal comfort of clothing. Studies $[2,7,8]$ contain laboratory results which could be used for research into the thermal characteristics of structural membranes, as well as the standards in the field of thermal protection (thermal resistance of air layers and surface convective resistance) [9].

The CFD procedure for the dynamic approach to calculating the energy balance was developed in the work of Devulder [10] and Harvie [6], who took into consideration the external environmental conditions, the required internal conditions and the properties of the material (the border layer between the external and internal environment).

This study focused on the determination of the thermal-physical properties of the border assembly consisting of two structural fabric membranes at a distance of $70 \mathrm{~cm}$. The air layer between the membranes is closed. According to the authors' findings, to date no experimental studies have been carried out on similar structures with an air layer $30 \mathrm{~cm}$ thicker, nor can any data be found in the literature which offers information on the thermal resistance of such a structure. The Membrane assembly thermal model [10] is necessary for an CFD analysis, which led the authors to conclude that by registering the air temperature inside and outside the dome, the temperature of the border surfaces, the air temperatures between the border surfaces, and solar radiation, they could by analytical means quantify the thermal resistance for the double membrane with an unventilated air layer. The results of comparative measurements on identical, neighbouring objects with a single and double closed fabric membrane, with an accompanying analysis, were shown in [11]. By implementing a stationary approach [12], the thermal behaviour of this specific border assembly 
consisting of an unventilated air layer and cylindrical fabric membranes was determined.

\section{THE METHOD AND INSTRUMENTS}

For the purpose of this study, a characteristic object with two independent halls was selected (Fig. 2), where one of the halls is covered by a single, and the other by a double fabric roof membrane (Fig. 1a). The object is located in Belgrade, in a part of the city known as Sumice (a residential area with large green surfaces). The hall was constructed for sports activities. Both halls have a $24 \times 44$ $\mathrm{m}$ in base, and are $11 \mathrm{~m}$, high (Fig. 1b). The shape of the halls is semi-cylindrical, without lanterns on the roof to allow for natural ventilation, and the main load-bearing construction is in the shape of an arch and made of glued laminated timber. The roof cover is a fabric membrane 0,8 mm thick, with a polyester core and PVC protection. The border assemblies consist of identical membranes located on the upper and lower side of the main load-bearing arches. The air enclosed between the double membrane, forms a closed air "pillow" $\delta_{\mathrm{m}}^{\mathrm{A}}=70 \mathrm{~cm}$ thick.

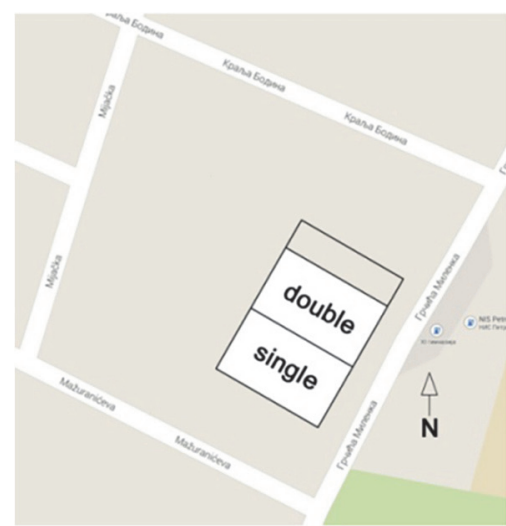

a.

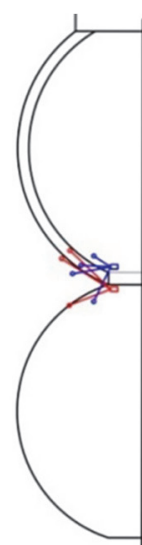

b., 11.00

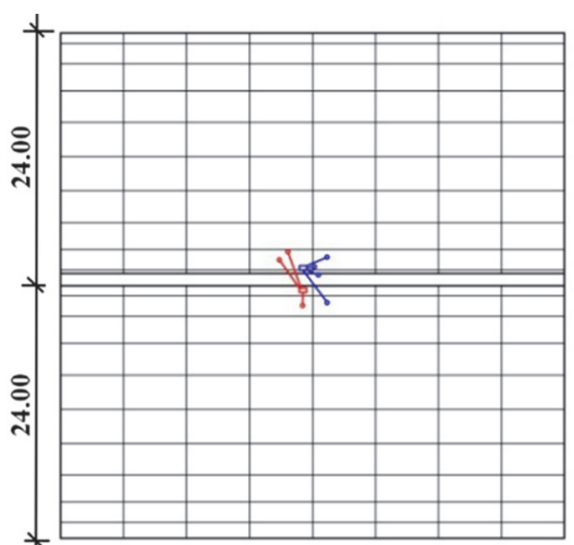

44.00

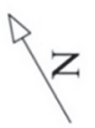

AIR TEMPERATURES 口 Data Logger - Probe SURFACE TEMPERATURES 口. Data Logger - Probe

Figure 1 a) Aerial plan; b) Plan and section of the building with probes placement
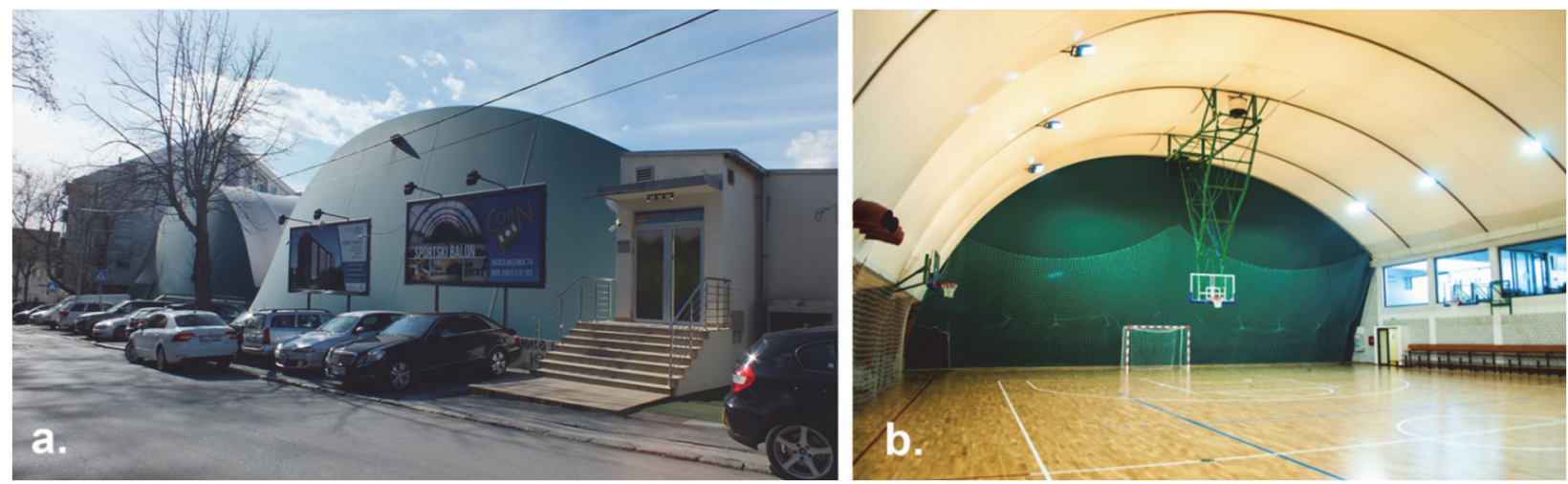

Figure 2 Double roof membrane hall: a) External view looking; b) Internal view looking

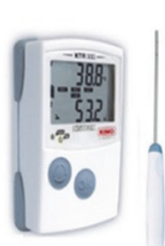

a. b.

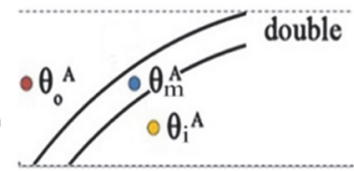

c.

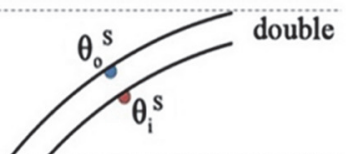

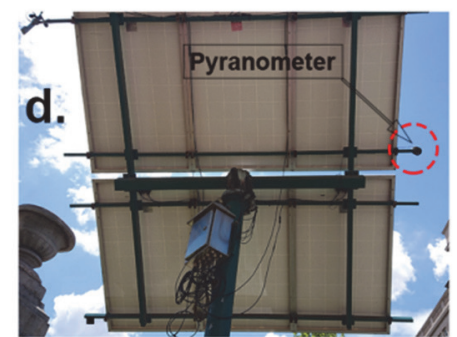

e.

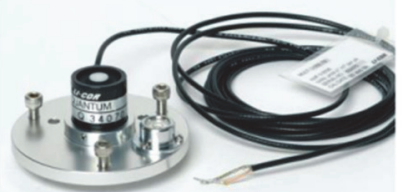

Figure 3 a) Kistock Data loggers with temperature wire probes; b) Position of air temperature probes; c) Position of surface temperature probes; d) Backside of inclined PV pane with measurement equipment; e) Li-Cor Solar radiation sensor

The measurements of the inside $\left(\theta_{\mathrm{i}}^{\mathrm{A}}{ }^{\circ} \mathrm{C}\right)$ and outside air temperature $\left(\theta_{\mathrm{o}}{ }^{\mathrm{A}}{ }^{\circ} \mathrm{C}\right)$, of the air layer between the membranes $\left(\theta_{\mathrm{m}}^{\mathrm{A}}{ }^{\circ} \mathrm{C}\right)$ and the surface temperatures of the inner $\left(\theta_{i}{ }^{\circ} \mathrm{C}\right)$ and outer membrane $\left(\theta_{\mathrm{o}}^{\mathrm{S}}{ }^{\circ} \mathrm{C}\right)$ were taken with the Kimo KTR 300, with original factory settings, with double channel DataLoggers and temperature wire probes Termistor type Pt100 class A as per IEC 751 with measuring range $-20{ }^{\circ} \mathrm{C}$ to $120{ }^{\circ} \mathrm{C}$ (Fig. 3a). The air temperature was measured with four probes, while the temperatures of the membranes were measured with three probes, whose positions are shown in Fig. 1b, 3b, 3c. The temperatures were measured every 30 minutes over a period from 20.6.2014 to 5.7.2014. The data were recorded on magnetic recording mediums. The accuracy of the probes was $\pm 0.4 \%$ of the value displayed or $\pm 0.3{ }^{\circ} \mathrm{C}$ according to the original factory settings for the measured temperature less than $100{ }^{\circ} \mathrm{C}$. 
The solar irradiance (Ig) data were measured using the equipment from the Laboratory for electro-energetic systems of the Faculty of Electrical Engineering in Belgrade at the Vukov monument location, at a distance of $2.5 \mathrm{~km}$ from where the hall is located. The measurements were made using a pyranometer: a Li-Cor silicon cell (Fig. $3 \mathrm{~d}$ and $3 \mathrm{e}$ ) fixed on a platform with Photo-Voltaic cells which automatically adjusts to the position of the sun, from June 19 to July 5, 2014. Pyranometer was calibrated against an Eppley Precision Spectral Pyranometer (PSP) under natural daylight conditions. Calibration uncertainty under these conditions is estimated as $\pm 3 \%$ typical, within $\pm 60^{\circ}$ angle of incidence.

In addition to the measurements carried out on the location itself, official data were used on the weather conditions during the analyzed period. These data were taken from the Ogimet measuring station WMO 13274 which is located $2 \mathrm{~km}$ north-west of the object (an automatic measuring station with a recording interval of 3 hours) and the Wunderground measuring station ISRBIJAB2, located approximately $2.5 \mathrm{~km}$, north-east of the object (with a recording interval of 5 minutes). The official data were necessary due to the wind speed and direction, and as a control mechanism of precision for the data obtained from the Kistock Data Loggers KTR300.

\section{THE RESULTS AND DISCUSSION}

The diagrams which represent a detailed account of all the officially measured air temperatures from the measuring stations WMO13274 $\left(\theta_{1}{ }^{\mathrm{A}}\right)$ and ISRBIJAB2 $\left(\theta_{2}^{\mathrm{A}}\right)$, the measured outer air temperatures above the object $\left(\theta_{\mathrm{o}}^{\mathrm{A}}\right)$, as well as the inner air temperature $\left(\theta_{\mathrm{i}}^{\mathrm{A}}\right)$, the air temperature between the membranes $\left(\theta_{\mathrm{m}}^{\mathrm{A}}\right)$ and the temperature of the membranes themselves $\left(\theta_{\mathrm{i}}^{\mathrm{S}}, \theta_{\mathrm{o}}^{\mathrm{S}}\right)$ are shown and analyzed in [11]. The results presented in [11] were compiled over a period of time when heating and ventilation systems were not in use.

The diagram which represents a detailed description of the official wind speeds recorded at the measuring station ISRBIJAB2, maximum wind gusts, as well as precipitation accumulation is shown in Fig. 4.

The diagram which represents the sun irradiance in the air in close proximity to the Vukov monument is presented in Fig. 5. A comparison of this diagram and the one in Fig. 4 indicates a connection between the decrease in sun radiation and periods of rain accumulation. A drastic decrease in irradiance and/or short-term oscillations with periods with no rain accumulation indicate heavy cloud coverage during these times.
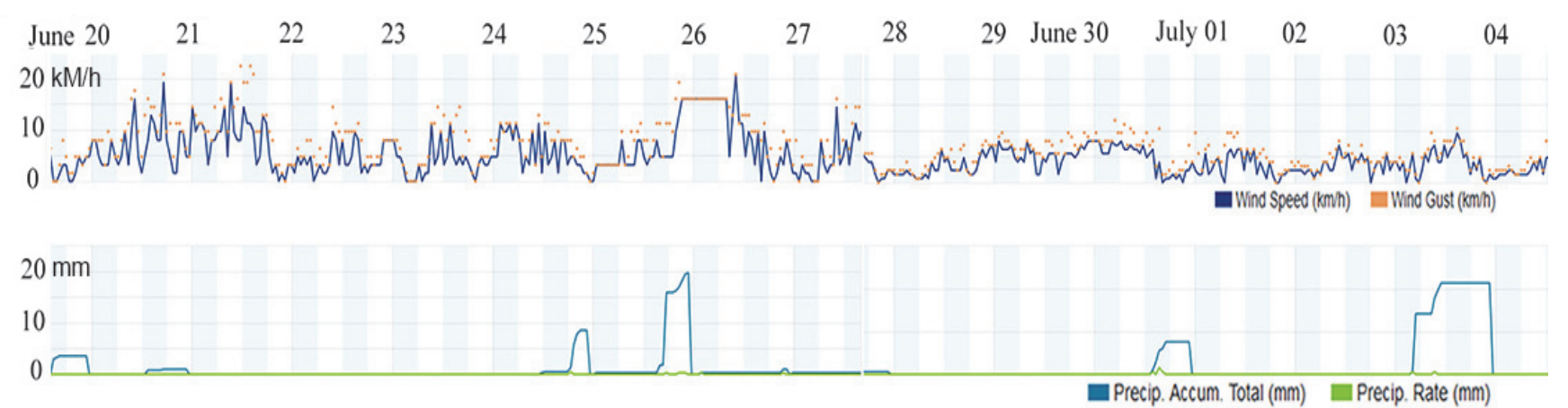

Figure 4 Official records: Wind speed and gusts / km/h, Rain accumulation / mm

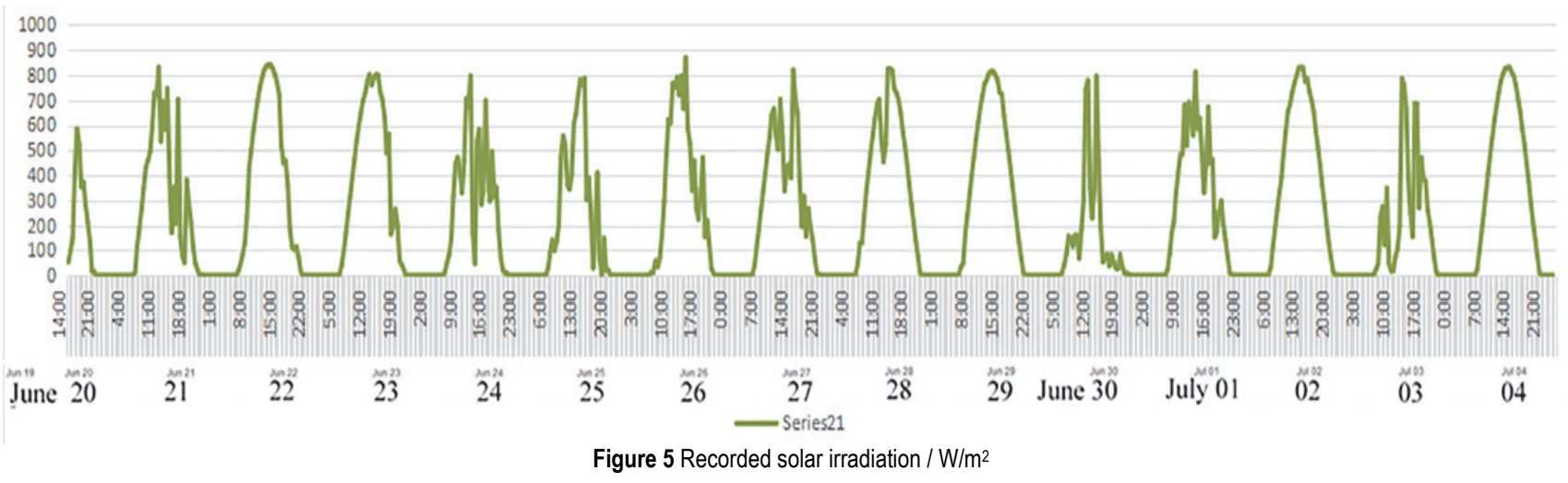

All of the compiled data indicate that the air temperatures inside these objects and the temperatures of the surfaces themselves are non-uniform. The thermal state of the outer membrane instantaneously reacts to the weather and climate variations in the outer radiation. The sun irradiance itself is changeable, resembling sinusoidal distribution during the day, and depends on the atmospheric conditions. Thus, the values of this parameter range from $0 \mathrm{~W} / \mathrm{m}^{2}$ to $830 \mathrm{~W} / \mathrm{m}^{2}$. The thermal state of the inner membrane during the day, while there is still a thermal flux from the irradiance downwards, indicates a constant negative difference compared to the outer membrane (the colder one). The exception is the moment when it begins to rain and there is a sudden drop in temperature of the outer membrane. A higher temperature is then recorded for the inner membrane, compared to the outer one, so the heat flux is in the opposite direction upwards.

The exchange of energy between the environment and the object during night time does not exist or it is focused 
upwards. Measurements have indicated approximately identical temperatures of the outer and inner membrane, while in the morning hours due to the "clear sky" effect, the inner membrane is negligibly warmer than the outer one.

These thermal phenomena are specific to tensile membrane structures primarily due to their negligible conduction (Fig. 6) and specific convection resistance, which in the case of double structures occur four times $\left(R_{\mathrm{Se}}\right.$
- thermal transmittance from the outer air onto the outer membrane surface, $R_{\text {Som }}$ - thermal transmittance from the outer membrane to the air between the membranes, $R_{\text {Sim }}$ thermal transmittance from air from the middle to the inner membrane, $R_{\mathrm{Si}}$ - thermal transmittance from the inner membrane to the inner air). They are also due to the stratification of the layers of large volume within the hall, which in the absence of a thermal flux and solar irradiance, leads to inverse occurrences.
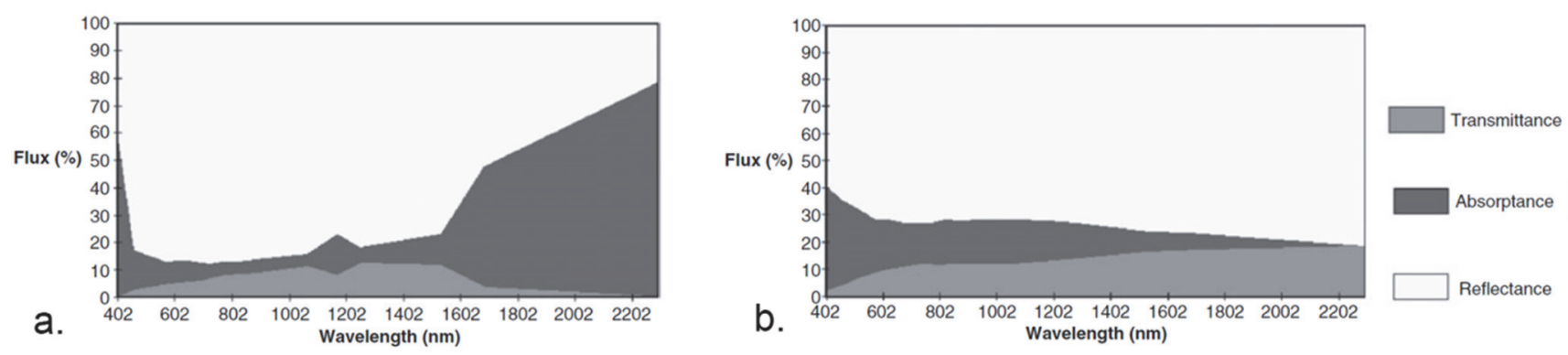

Figure 6 Solar optical properties of membranes [10]: a) PVC coated polyester; b) PTFE coated fibreglass

The key events which need to be modelled prior to any attempts at a simulation of the thermal behaviour of closed membrane space on an annual level are certainly the outer air temperature, inner air temperature, rain accumulation, wind, solar irradiation.

\section{AIR LAYERS INFLUENCE ON ASSEMBLIES BUILT IN}

The air layers in assemblies have thermal resistance which is regulated by norms [9]. The aforementioned standards determine the calculation methods for thermal resistance and the coefficient of thermal conduction. The air layer is limited by two surfaces which are in essence flat, parallel to one another and approximately perpendicular to the direction of the thermal flux with an emissivity which is greater than 0.8 .

The currently available literature on physics in civil engineering includes a study [12] on the thermal-physical properties of vertical and horizontal walls with an enclosed air layer within the wall made of solid material. The results for the thermal resistance of the air layer are shown in Fig. 7 [9].

According to the EN ISO 6946:2007, SRPS ISO 6946:2005 and DIN - 4701 standards, equivalent thermal resistance of unventilated air layers depending on the thickness of the layer and the direction of the thermal flux, with high surface emissivity (e), is shown in Fig. 7.

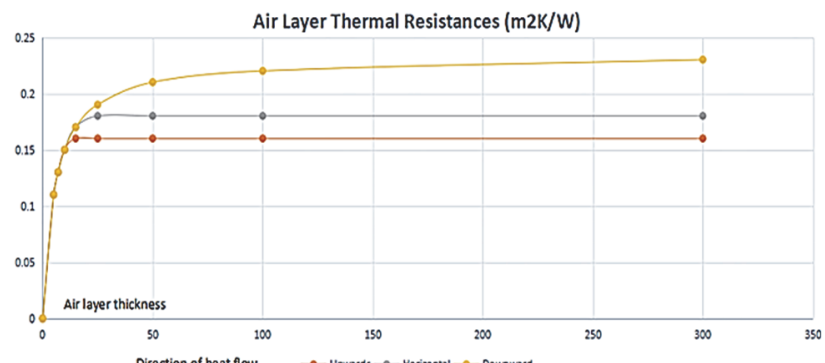

Figure 7 Thermal resistance coefficients through an unventilated air layer with emissivity greater than 0.8

A special calculation procedure defined by the standards (EN ISO 6946:2007, SRPS ISO 6946:2005) is applied when there are no tables of values. The thermal resistance of the studied air layer calculated by that procedure for the summer months would be $R_{\mathrm{g}}=0.1732$ $\mathrm{m}^{2} \mathrm{~K} / \mathrm{W}$.

Research in the field of design of ready-to-wear clothing with two-layer fabric assemblies was carried out with the purpose of achieving better thermal-physiological comfort of the users in warmer weather conditions. Research [7] was carried out in laboratory conditions with fourteen different outside samples and two different inner samples, that is, 28 combinations of fabric were studied using the Alambeta measuring instrument [8]. All of the samples were cotton fibre or a combination of polyester and cotton, or polyester and viscose. The thickness and unit weight of the selected fabrics were 3 to 4 times smaller than the structural polyester membranes covered in PVC, but the thermal resistance coefficients of the air layer in all the studied samples were within the range of 0.14 to 0.25 $\mathrm{m}^{2} \mathrm{~K} / \mathrm{W}$. The studied combinations of fabric had air gaps of $5 \mathrm{~mm}, 7 \mathrm{~mm}$ and $10 \mathrm{~mm}$, and the results are sublimated in the diagram shown in Fig. 8.

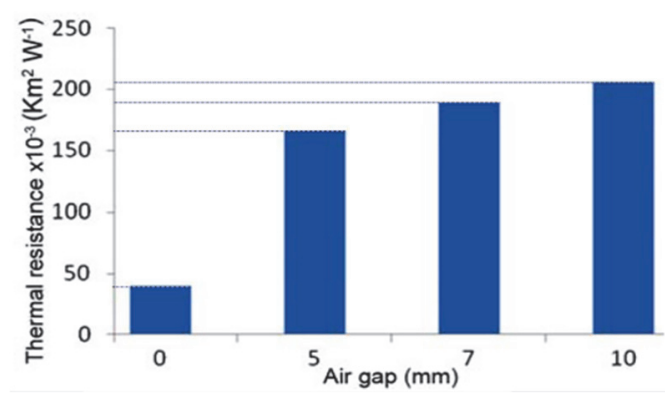

Figure 8 Effect of different air gaps on thermal resistance of two-layer fabric assemblies

It is clear that the results do not take into consideration the transfer of energy from the air by convection, since with the use of the Alambeta instrument, the thermal energy is transferred through direct contact. Furthermore, what can also be noted is the regular quick increase in the thermal resistance of air gaps up to a thickness of $10 \mathrm{~mm}$ by the second order transfer function, while the expectation 
was that thicker layers will have a more constant thermal resistance which will not depend on the thickness of the air layer.

\section{THERMAL RESISTANCE OF THE AIR LAYER BETWEEN TRANSPARENT FABRIC ROOF MEMBRANES}

In this paper we studied the influence of transparent, lightweight, thin, double fabric material on the thermal resistance of an air layer with a convex wall geometry (a curved, semi-cylindrical surface), which is closed unventilated, $70 \mathrm{~cm}$, thick, since the standards [9] only take into consideration flat horizontal and vertical surfaces at an angle of up to $30^{\circ}$.

The known parameters which influence the thermalphysical properties of the enclosed air, with all the properties were included into the calculations in order to use the measured temperatures to quantify the overall thermal resistance $R_{\mathrm{T}}$. The compiled data described in section 3 were used in the analytical model.

The thermal resistance was calculated through a multilayered semi-cylindrical wall, within an assumed stationary state. The air layer within the semi-cylindrical wall was not solid, and the included coefficient air conduction $\lambda_{\mathrm{m}}{ }^{\mathrm{A}}$ has a value which is a consequence of radiation, the intensity of the re-circulation of air within the layer and the conductive transport of heat through the air. The calculations were made based on the measured parameters.

The hall is semi-cylindrical in shape, and has the aforementioned dimensions. It is considered an ideal semicylinder since the surfaces of its lateral sides are quite smaller compared to the surface of the roof, so their influence can be approximated sufficiently well to an ideal semi-cylinder. The intensity of radiation $I_{\mathrm{g}} \mathrm{W} / \mathrm{m}^{2}$ was measured in the immediate vicinity of the object itself, and is shown in Fig. 5. All of the measured temperatures, of the outer air, inner air, and the outer surface and inner membrane, as well as the thermal flux from the solar irradiance focused downwards were taken into consideration when calculating the thermal characteristics. In order to include radiation as an influential factor in the thermal calculations, in addition to the measured outer temperatures, calculations of fictive solar-air temperatures were done $\left(\theta_{\text {sol-air }}{ }^{\circ} \mathrm{C}\right)[12]$.

The solar-air temperature represents a fictive temperature which the outside air should have in order to cause a thermal flux on the surface of the wall, equal to the one which originates from the actual influence of sunlight and outside temperature. In other words, if the outside air temperature had the value of the sunlit air temperature, then the outer side of the outer membrane would be heated through convection to the same temperature as in the actual conditions when the outside temperature and sunlight have a simultaneous effect [12]. It was necessary to calculate this temperature in order to apply the standard stationary approach to thermal calculations of the thermal resistance coefficient for a multi-layered semi-cylindrical wall, that is, to include the influence of the sun radiation in the calculation model indirectly. The outside and inside thermal surface convection coefficients $\left(R_{\mathrm{se}}, R_{\mathrm{si}}\right)$ were averaged along the entire surface, and the values were taken into consideration in accordance with the thermaltechnical standards [9] along with the speed of the outside air flow (Fig. 4). Velocity of recirculation flow of closed air between membranes was not measured. Certainly, all heat transfer models are present in this case (radiation, convection, conduction). Overall approach was conducted with assumed simplifications in sense of engineering usability, the process was calculated in the manner of standard wall layer calculation where calculated air conduction actually incorporates the other two influences of convection and radiation. The movement of indoor air (inside hall) was taken into account by assumption that velocity does not exceed the $1.5 \mathrm{~m} / \mathrm{s}$ defined by indoor air thermal comfort regulations, and acquired convective coefficient was $10 \mathrm{~W} / \mathrm{m}^{2} \mathrm{~K}$ for inside membrane to indoor air.

Sol-air temperatures were calculated using Eq. (1):

$\Theta_{\text {sol-air }}=\Theta_{\mathrm{o}}^{\mathrm{A}}+\frac{a \cdot I_{\mathrm{g}}-e \cdot \Delta G}{\alpha_{20}^{\mathrm{S}}},{ }^{\circ} \mathrm{C}$

where: $a=15 \%$, absorption coefficient of the membrane; $e=0.86$, long wave emissivity; $\alpha_{20} \mathrm{~S}$ - convection coefficient of the outside surface, at daily average wind speed; $\Delta G=-3.7^{\circ} \mathrm{C}$, correction coefficient for the used coefficients $\alpha_{20} \mathrm{~S}$ and $e$, for Belgrade.

The thermal calculation for the semi-cylindrical surface, with a radius $r_{1}$ or $r_{2}$ (Fig. 10b), takes into consideration the borderline conditions of the third degree by applying integrals in the equation for the thermal gain of a semi-cylindrical surface Eq. (2), that is, the equation for the linear thermal flux of a cylindrical surface Eq. (3) [13].

$$
\begin{aligned}
& Q=-\lambda \cdot A \cdot \frac{\mathrm{d} \Theta}{\mathrm{d} r}, \mathrm{~W} \\
& \frac{Q}{l}=q=-\lambda \cdot r \cdot \pi \cdot \frac{\mathrm{d} \Theta}{d r}, \mathrm{Wm}^{-1}
\end{aligned}
$$

By developing the aforementioned equations, we obtain the equation for the thermal flux of a cylindrical surface Eq. (4) and Eq. (5):

$$
\begin{aligned}
& q=\frac{\Theta_{\mathrm{o}}^{\mathrm{S}}-\Theta_{\mathrm{i}}^{\mathrm{S}}}{\frac{1}{\lambda \cdot \pi} \cdot \ln \left(\frac{r_{2}}{r_{1}}\right)}=\frac{\Theta_{\mathrm{o}}^{\mathrm{S}}-\Theta_{\mathrm{i}}^{\mathrm{S}}}{R_{\mathrm{memb}}}, \mathrm{Wm}^{-1} \\
& q=\frac{\Theta_{\mathrm{o}}^{\mathrm{A}}-\Theta_{\mathrm{i}}^{\mathrm{A}}}{\frac{1}{\alpha \cdot \pi \cdot r}}=\frac{\Theta_{\mathrm{o}}^{\mathrm{A}}-\Theta_{\mathrm{i}}^{\mathrm{A}}}{R_{\mathrm{S}}}, \mathrm{Wm}^{-1}
\end{aligned}
$$

By indicating that (4) and (5) are equal, separating thermal resistance $R$ based on the adopted model (Fig. 9b), the following Eq. (6) is obtained:

$$
q=\frac{\Theta_{\text {sol-air }}{ }^{\mathrm{A}}-\Theta_{\mathrm{i}}^{\mathrm{A}}}{R_{\mathrm{Se}}+R_{\mathrm{memb}}+R_{\mathrm{m}}^{\mathrm{A}}+R_{\mathrm{memb}}+R_{\mathrm{Si}}}=\frac{\Theta_{\mathrm{O}}^{\mathrm{S}}-\Theta_{\mathrm{i}}^{\mathrm{S}}}{R_{\mathrm{memb}}+R_{\mathrm{memb}}}(6)
$$


By solving the equation according to $R_{\mathrm{m}}{ }^{\mathrm{A}} \mathrm{mK} / \mathrm{W}$ the linear conduction resistance through the air layer enclosed between the membranes and the further extraction of the fictive coefficient of conduction $\lambda_{\mathrm{m}}{ }^{\mathrm{A}}$ from the value of $R_{\mathrm{m}}{ }^{\mathrm{A}}$, we obtain Eq. (7) for the value of the fictive coefficient of conduction for the air layer, which in reality is the function of radiation, convection and conduction.

$\lambda_{\mathrm{m}}{ }^{\mathrm{A}}=\frac{1}{R_{m}{ }^{A} \cdot p} \cdot \ln \left(\frac{r_{2}+\delta_{\mathrm{memb}}}{r_{1}-\delta_{\mathrm{memb}}}\right), \mathrm{Wm}^{-1} \mathrm{~K}^{-1}$

By applying the equations the sol-air temperature was calculated (Eq. (1)), core conductivity $\lambda_{\mathrm{m}}{ }^{\mathrm{A}}[14]$ and the thermal resistance $R_{\mathrm{g}}{ }^{\mathrm{A}}=\delta_{\mathrm{m}}{ }^{\mathrm{A}} / \lambda_{\mathrm{m}}{ }^{\mathrm{A}} \mathrm{m}^{2} \mathrm{~K} / \mathrm{W}$ of a semi- cylindrical non-ventilated air layer enclosed between fiber structural membranes (Fig. 10).

The calculation included all the dynamic changes of the solar radiation and the atmospheric conditions (cloud coverage, rain accumulation and wind) during the day, which led to sudden changes in the parameters which were used in the calculations. In cases when a very small or negative difference was noted between the outer and inner air temperature, as well as the temperature of the outer and inner membrane, the calculated values of thermal resistance did not have a real value and were not taken into consideration.

The average calculated value of thermal resistance for the air layer is $R_{\mathrm{g}}{ }^{\mathrm{A}} 0.225 \mathrm{~m}^{2} \mathrm{~K} / \mathrm{W}$.

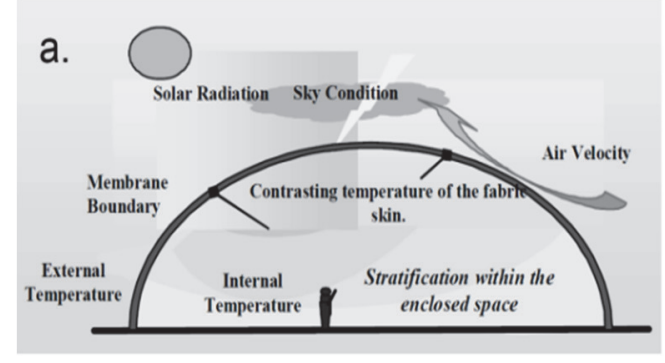

b.

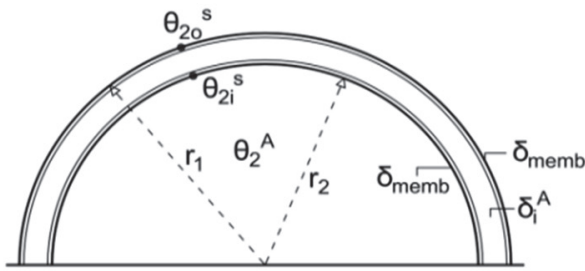

C.

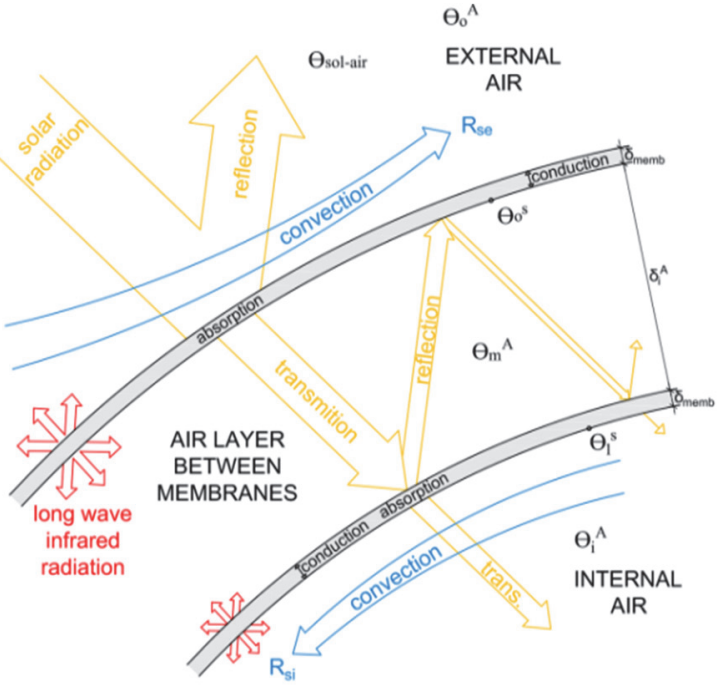

Figure 9 a) Overall environmental influence on the membrane enclosed spaces; $b$ ) The idealized cross-section of the hall; $c$ ) Thermal transmittance through the model with a double fabric membrane

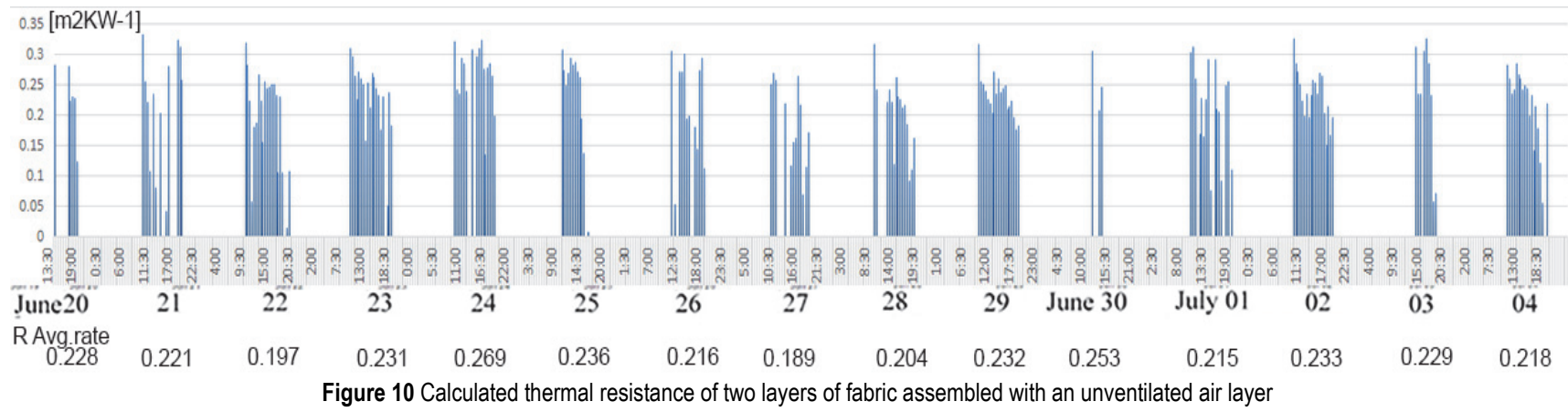

\section{RESULT ANALYSIS}

The selected methodological approach required that a real object be used to compile the necessary parameters (solar irradiance, outer air temperature, the temperature of the border layers of fabric membranes, inside air temperature, wind speed) and the values of the initial thermal-physical properties needed for the application of a stationary approach to calculating thermal resistance. This included all the forms of heat transfer and analyzed the influence of a two-layer membrane assembly with an unventilated air layer (Fig. 9a). The obtained results for thermal resistance are shown in Fig.10, and are based on a stationary approach which includes the familiar thermal flux, which in this case originates from the solar irradiance and is focused downwards. It is clear that the thermal behavior of the membrane structure is non-uniform and that it depends on the measured parameters.

The transmittance of solar radiation through an outer surface into an unventilated gap between the membranes (4\% to $10 \%$ flux [7]), long wave infrared radiation (approximately 7\% flux [7]) and reflectance from the lower border surface were not take into consideration by the standards, while it is evident when the outside air temperature exceeds $30^{\circ} \mathrm{C}$ and wind speed greater than 4 $\mathrm{m} / \mathrm{s}$ lead to a decrease in thermal resistance (Fig. 11a). Passing cloud coverage, changes in rain accumulation, wind and decreased solar irradiance caused changes in the temperature of the outer membrane which during some periods of the day was smaller than the temperature of the 
inner membrane. The reason for such a state is the expressed stratification of the inner air and the convective thermal resistance from the membrane to the air, which led to a thermal flux upwards and results with no real values.

The analysis of ready-made fabrics [7] and the existing standards [9] of thermal resistance (Fig. 8) of air layers of various thickness were treated as constants, while analytic means were used to determine that there were changes

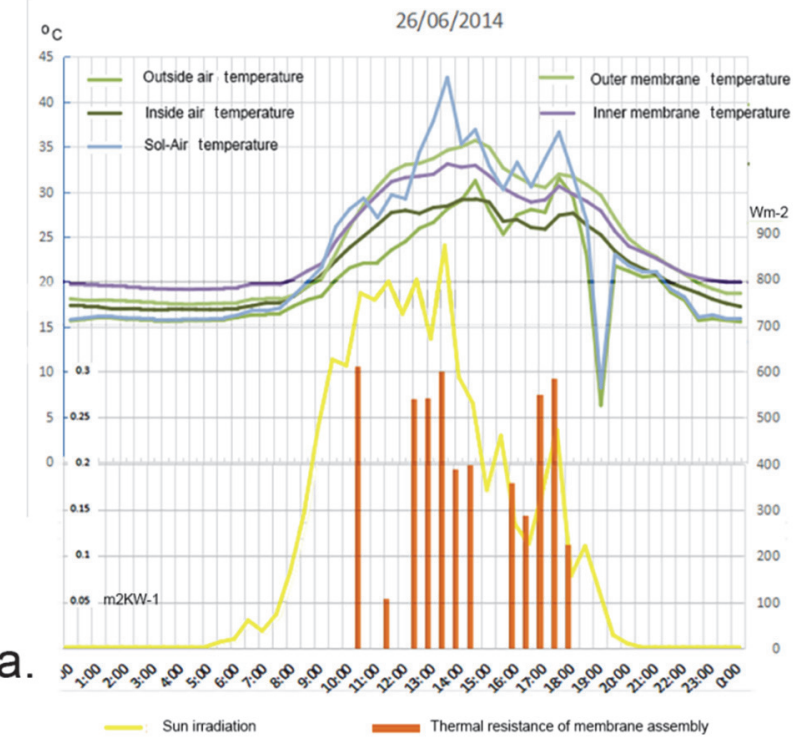

depending on solar irradiance as a local factor, current climatic conditions, as well as the thermophysical and optical characteristics of the fabric itself (Transmittance, Reflectance and Shading coefficient). The topology of the surface and its position in relation to the solar path directly influence the amount of solar energy which affects objects of this kind, which also causes a stratification of the layers within the object.

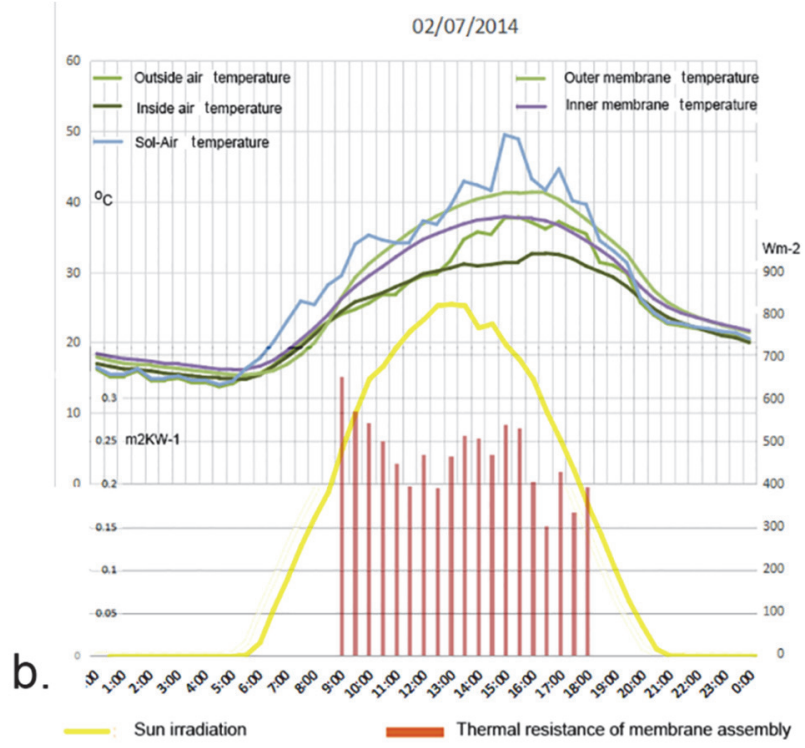

Figure 11 Calculated thermal resistance of double membrane assembly in relation to measurements: a) Summer cloudy day (26/06/2014); b) Summer clear day $(02 / 07 / 2014)$

\section{CONCLUSION}

The poor thermal resistance of these structures is one of the main reasons against the more widespread application of covers for closed spaces due to the increased energy consumption for heating/cooling. One of the ways of improving the energy efficiency of these objects is the implementation of a double fabric roof membrane structure with an enclosed non ventilated air layer.

The results of this study differ compared to laboratory results. It has been indicated that the actual thermal insulation properties of closed air layers on the constructed object, compared to the standardized non-uniform values, depend on solar irradiation and all the climatic factors which affect the structure at a given moment. The average value of thermal resistance is greater than the standardized one, but it could in many ways differ for various parts of the surface, which due to the topology of the surface are in semi-shade or shade due to decreased thermal flux. The increased values in the thermal resistance can be explained by the transmittance of long-wave IR sun radiation through upper transparent fabric membrane and the reflectance of the radiation from the bottom border surface, which was not fully taken into consideration in the standards, and which leads to increased thermal resistance compared to the standardized values during the summer months.

The contemporary approach to thermal behavior of closed double fabric structures is rendered possible by using the Computer Fluid Dynamics (CFD) model $[2,6]$, but to this day a Double membrane construction model has not been formed.
The shown equations can be used to predict the resistance of a semi-cylindrical air layer enclosed between two membranes, and to optimize the thermal comfort during hotter weather conditions by applying CFD modelling, using results of this study.

Further research can focus on the study of the thermalphysical properties of the layers of air enclosed between three-layered membranes, or layers which might have better reflectance features.

\section{Acknowledgment}

This paper is part of the research in the scientific research project TR36045 "Revitalization of preschool buildings in Serbia: Program and Methods of environmental, functional and energy efficiency improvement", funded by the Ministry of Education, Science and Technological Development of the Republic of Serbia during the period 2011/2014 (2020).

\section{REFERENCES}

[1] Karwath, M. (2011). Membrane Structures with Improved Thermal Properties. International Conference on Textile Composites and Inflatable Structures. Barcelona, 312-318.

[2] Zeinab, S. et al. (2006). Textile fabrics as thermal insulators, Autex Research Journal, 6(3), 148-161.

[3] He, J. \& Hoyano, A. (2009). Measurement and Simulation of the Thermal Environment in the Built Space under a Membrane Structure. Building and Environment, 44, 11191127. https://doi.org/10.1016/j.buildenv.2008.08.003

[4] He, J. \& Hoyano, A. (2010). Measurement and Evaluations of the Summer Microclimate in the Semi-enclosed Space 
under a Membrane Structure. Building and Environment, 45, 230-242. https://doi.org/10.1016/j.buildenv.2009.06.006

[5] Elnokaly, A., Chilton, J., \& Wilson, R. (2003). Environmental Performance of Spaces Enclosed or Semienclosed by Fabric Membrane Structures. Textile Composites and Inflatable Structures, 171-176.

[6] Harvie, G. N. (1995). An Investigation into the thermal Behaviour of Spaces Enclosed by Fabric Membranes. $\mathrm{PhD}$ thesis, Cardiff University of Wales, Cardiff, UK.

[7] Deepti, G., Ashis, S., \& Sunil, K. (2013). Thermal Properties of single and Double Layer Fabric Assemblies. Indian Journal of Fiber \& Textile Research, 38, 387-394.

[8] Devanand, U. (2012). Objective Measurement of Heat Transport through Clothing. International Journal of Engineering Research and Development, 2(12), 43-47.

[9] EN ISO 6946/2007, SRPS ISO 6947/2005, DIN 4701.

[10] Devulder, T., Wilson, R., \& Chilton, J. (2015). The Thermal Behaviour of Buildings Incorporating Single Skin Tensile Membrane Structures. International Journal of Low Carbon Technologies, 2, 195-213. https://doi.org/10.1093/ijlct/2.2.195

[11] Kostic, D., Milosevic, V., Bogdanovic, V., Vasov, M., Vucur, A. (2018). Influence of Single and Double Membrane Roofs on Thermal Behaviour of Enclosed Space. Technical Gazette, 25(1), 188-196. https://doi.org/10.17559/TV-20161205160405

[12] Todorović, B. (2005). Climatisation 2. Belgrade, Serbia. SMEITS.

[13] Djordjevic, B. et al. (2005). Thermodynamics and thermos techniques. Belgrade, Serbia: Technological Faculty.

[14] Reyer, E. (2001). Bauphysik. Bochum, Germany. Lehrstuhl und Institut fur Baukonstruktionen, RUHR-Uni.

\section{Contact information}

Dragan KOSTIC, PhD, Associate Professor

(Corresponding author)

University of Nis, Faculty of Civil Engineering and Architecture,

A. Medvedeva 14, 18000 Nis, Serbia

E-mail: dragan.s.kostic@gmail.com

Veliborka BOGDANOVIC, PhD, Full Professor

University of Nis, Faculty of Civil Engineering and Architecture,

A. Medvedeva 14, 18000 Nis, Serbia

E-mail: veliborka.bogdanovic@gaf.ni.ac.rs

Marko Ilic, MSc Mech.Eng., Teaching Assistent

University of Nis, Faculty of Mechanical Engineering,

A. Medvedeva 14, 18000 Nis, Serbia

E-mail: marko.ilic.me@gmail.com

Miomir VASOV, PhD, Associate Professor

University of Nis, Faculty of Civil Engineering and Architecture

A. Medvedeva 14, 18000 Nis, Serbia

E-mail: miomir.vasov@gaf.ni.ac.rs

Vuk MILOSEVIC, PhD, Assistant Professor

University of Nis, Faculty of Civil Engineering and Architecture,

A. Medvedeva 14, 18000 Nis, Serbia

E-mail: vukamer@yahoo.com 